\title{
The Relationship of Chronic Diseases and Health Status to the Health Services Utilization of Older Americans
}

\author{
Caroline S. Blaum, Jersey Liang, ${ }^{\dagger \neq}$ and Xian Liu ${ }^{\ddagger}$
}

OBJECTIVE : To study simultaneously the relationships among chronic diseases and physical health status as they affect health services utilization of older adults.

DESIGN: Secondary analysis of a large, cross-sectional health interview survey, the Supplement on Aging of the 1984 National Health Interview Survey, using multiple equation methods to evaluate disease-specific impacts on physical health status, the direct impact of specific diseases on utilization of physician services and hospital care, and the indirect impact of specific diseases on utilization, mediated through physical health status.

PARTICIPANTS: A total of 11,497 people aged 65 and older, representing a complex, multistage sample of the noninstitutionalized, older adult population of the United States.

MEASUREMENTS: Predictor variables included specific chronic diseases (hypertension, arthritis, diabetes, cancer, and atherosclerotic heart disease), self-rated health status, and total number of disabilities. Control variables included age, gender, race, education, social integration. Outcome variables were physician visits and hospital stays.

MAIN RESULTS: It was shown that different diseases have different relative impacts on physical health status, probability of utilization, and amount of utilization, if any, and different chronic diseases have a different mix of direct and indirect effects on utilization.

CONCLUSION: The impact of chronic disease on health services utilization in a community-dwelling population is not a simple or direct relationship. Diseases vary according to their impact on different types of utilization, their impact on the probability of any health services use versus the amount of use, and on how much their effect on utilization is mediated through health status. J Am Geriatr Soc 42:1087-1093, 1994.

From the "Division of Geriatric Medicine, Department of Internal Medicine, University of Michigan Hospitals; tInstitute of Gerontology; and \$School of Public Health, University of Michigan, Ann Arbor, Michigan.

This research was supported by the Public Health and Aging Training Grant, NIA:2-T32-AG00134, GRTC Pilot Project Grant, University of Michigan Geriatrics Center, NLA:SP30 AG08808-04, NRSA Individual Postdoctoral Award, NIA:1-F32 AG05633, The John A. Hartford Foundation Academic Research Training Program, and the Ann Arbor Veterans Administration Medical Center Geriatric Research, Education, and Clinical Center.

Address correspondence to Caroline S. Blaum, MD, University of Michigan Medical School, Division of Geriatrics, 300 North Ingalls Street, NI3A00, Ann Arbor, MI 48104-0405.
Orta hronic diseases lead to disabilities, affect the health status of millions of older Americans, and are responsible for increasing health services utilization. ${ }^{1}$ Although many researchers have studied direct links from disability and health status to health services utilization, or from chronic diseases to disability and health status, these efforts were largely based on single equation models and have given us only a piecemeal understanding of chronic diseases and their impacts on health services utilization. To understand the whole picture, it is important to study simultaneously the relationships among chronic diseases, disability, and health status as they affect health services utilization. We used multiple equation ${ }^{2}$ and two-stage ${ }^{3}$ modeling techniques to study these simultaneous linkages in a community-based population, and focused on the impact of specific chronic diseases. Such an approach has led to two important contributions to the understanding of health service utilization of older Americans: (1) how specific chronic diseases affect physical health status, which mediates the impact of chronic diseases on utilization, and (2) insight into disease-specific patterns of direct and indirect (mediated by physical health status) effects on utilization.

Our research goal was to evaluate the hypothesis that there is a causal pathway from chronic diseases to disability and self-rated health status (SRHS), and that physical health status (which includes chronic disease, disability and $\mathrm{SRHS}^{4}$ ) is an important determinant of health services utilization. We first studied the impact of specific chronic diseases on disability and SRHS to estimate the relationships among the determinants of physical health status. We then studied the direct impact of specific chronic diseases on different measures of health services utilization, hospital stays, and physician visits in a community-based sample of older Americans. Finally, we estimated the indirect effects of chronic diseases on utilization, which gave us an empirical estimate of how much the effects of different chronic diseases on utilization are mediated by physical health status.

\section{METHODS}

\section{Conceptual Framework}

The conceptual framework for this research uses two major models in health sociology to focus our study of disease-specific outcomes. The first model is the structure of physical health, which some researchers have viewed as a multidimensional construct involving chronic disease measures, disability measures, and self-rated health status. ${ }^{4}$ This conceptualization has been useful in modeling the interrelationships among chronic disease, disability, and health status 
and studying how these links are affected by exogenous factors such as gender, age, ethnicity, and social support. Although chronic disease is always shown to be important in this model, a disease-specific focus has not been evaluated, nor has the structure of physical health been studied for its impacts on health services utilization.

The second model is the Anderson Health Behavior Model of Utilization (HBM), a "patient-based" model used to study the determinants of people's health services utilization behavior. This model considers people's "need" (including disability, health status, and disease measures), "predisposing" (including health beliefs and attitudes), and "enabling"(including insurance and socioeconomic status) characteristics as important predictors of their use of health services. A consistent finding in such studies is the overwhelming importance of "need" variables in explaining utilization variance. ${ }^{6}$ Often disability and self-rated health status (SRHS) are used as measures of need. ${ }^{7-11}$ However, the relationship among the various components of "need," chronic diseases, disability, and health status, has not been addressed, nor has a disease-specific focus been commonly adopted.

We developed a conceptual model relating physical health status (ie, chronic diseases and their links to disabilities and self-rated health status) to health services utilization. Figure 1 shows a schematic diagram of our model, which incorporates a multidimensional structural model of physical health status as a predictor of utilization. As shown in the diagram, chronic diseases (represented in aggregate in Figure 1) act directly to predict disability, SRHS, and utilization. In addition, chronic diseases impact utilization indirectly, through effects on disability and SRHS. As Figure 1 shows, disability and SRHS intervene between chronic disease and utilization, illustrating our hypothesis that chronic diseases exert some of their effects on utilization through their impacts on disability and SRHS. Other important variables, such as demographic and socioeconomic characteristics, are considered to have direct effects on

Figure 1. Figure 1 is a simplified illustration of our conceptual model of the effects of physical health status on health services utilization. Chronic diseases (considered in aggregate for simplification) disability, and self-rated health status (shaded) are components of physical health status. All three have direct effects on health services utilization. However, chronic diseases act indirectly on health services utilization through effects on disability and self-rated health status. Disability also acts indirectly on utilization through effects on self-rated bealth status.

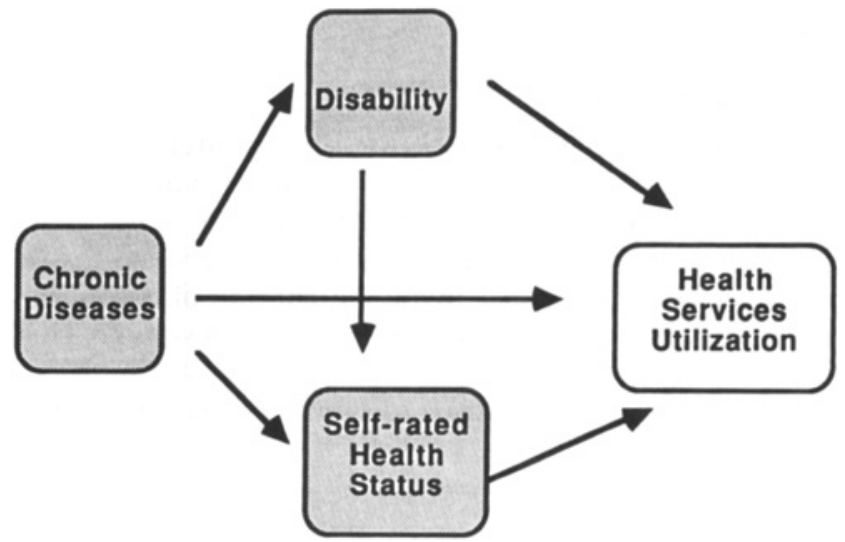

disability, SRHS, and utilization. As the arrows in Figure 1 show, our conceptual model specifies unidirectional relationships among predictor variables and outcome variables (recursive model).

\section{Data}

The data for these analyses are from the Supplement on Aging (SOA) of the 1984 Health Interview Survey (HIS). The SOA, a joint project of the National Center for Health Statistics and the National Institutes on Aging, is a complex, multi-stage sample of the noninstitutionalized population of the United States aged 55 to 74. The SOA sample consisted of half of the 1984 HIS respondents between the ages of 55 and 65 and all of the respondents aged 65 to 74 ( $n=$ $16,148)$. SOA respondents were given additional interviews focusing on health status, function, living situation, social support, and health services utilization. The details of how HIS participants were selected for the SOA, and issues regarding the complex sampling methodology, proxy respondents, and nonresponse have been well described.7, 12

\section{Variable Specification}

The variables of interest for this research included predictor variables, outcome variables, and control variables (Table 1). The SOA has 18 variables representing chronic medical conditions. We studied the most common chronic diseases: hypertension, diabetes mellitus, arthritis, cancer, and atherosclerotic heart disease (ASHD). The first four of these variables were based on responses to the corresponding SOA item. Representing heart disease presented more of a problem, because five to seven medical condition questions potentially refer to heart disease. We considered yes answers to the questions about angina, coronary heart disease, or myocardial infarction as representing the presence of atherosclerotic heart disease (ASHD). Nonspecific or other heart diseases, such as rheumatic heart disease, rheumatic fever, other heart attack, and hardening of the arteries were grouped with the remaining medical conditions into a summed variable, "other conditions," which was then di-

\begin{tabular}{l}
\hline Table 1. Variables studied \\
\hline Predictor Variables \\
Hypertension \\
Diabetes \\
ASHD \\
Cancer \\
Arthritis \\
Other diseases \\
Control Variables \\
Age \\
Gender \\
Race \\
Education \\
Social isolation \\
Outcome Variables \\
Self-rated health status \\
Number of disabilities \\
Physician visits per year \\
Hospital days per year \\
\hline
\end{tabular}


chotomized to indicate the presence of any of these other conditions.

Disability and SRHS were analyzed as both outcome and predictor variables in our model. The disability variable was the sum of the affirmative answers to the 24 disability variables available in the SOA. These variables include six activities of daily living, ${ }^{13}$ six instrumental activities of daily living, ${ }^{14}$ and 12 physical disability indicators, such as ability to lift 25 pounds, or ability to stand 2 hours.

Self-rated health was measured by one question in the SOA which asks, "How would you rate your overall health?" Answers are coded from 1, excellent, to 5, poor.

We developed models predicting two different types of health services utilization, physician visits and hospital stays. We used the self-reported number of physician visits and hospital stays in the past year as our variables. Although the Medicare-linked information was available for the sample, we chose to use self-reported utilization in order to parallel the other self-report data used in our analyses.

Demographic and social variables were controlled in all the statistical models used in this study. Age has a strong direct relationship with disability, ${ }^{1,15-17}$ and health services utilization. ${ }^{7}$ We chose as our analytic sample those 65 and older because this group is Medicare eligible, and we modeled age as a continuous variable. Statistically significant gender, racial, and socioeconomic differences have also been noted in disability, ${ }^{16-18}$ self-rated health, ${ }^{19}$ and utilization. ${ }^{17}$ Therefore, we controlled for gender, race, (white and nonwhite), and educational level in all our analyses, and we controlled for the absence of social isolation by the variable "talking to relatives once a week." Income was not chosen as a variable because it is missing in nearly $20 \%$ of cases and may be dependent on household composition. ${ }^{20}$ Educational level was chosen as an alternative indicator of socioeconomic status.

\section{Data Analysis}

The univariate characteristics of the variables used were examined to evaluate variable distribution and missing values. There are 11,497 respondents aged 65 or older in the SOA. We excluded respondents with missing data in any of our model variables, which resulted in approximately $11 \%$ dropped cases. The missing values were distributed among the variables in the models, with the most in any one variable at about $2 \%$. We did not correct for the complex sample design in the subgroup we analyzed. Because this will cause underestimation of variance, we required significance to be $P$ $<0.001$ except where otherwise discussed.

The same predictor variables were entered into all of the statistical models used. The five major chronic diseases (arthritis, ASHD, cancer, diabetes, and hypertension) were coded as dummy variables. Age, race, gender, educational level, social isolation, and presence of other comorbid diseases were entered as control variables.

In order to assess how specific chronic diseases affect the structure of physical health, we constructed multiple equation models. Two ordinary least square (OLS) regression models were used to study the direct effects of chronic diseases (and control variables) on disability and SRHS. These two models were hierarchical, that is, the control variables and chronic diseases were used to predict disability, and the control variables, chronic diseases, and disability were used to predict SRHS. The regression coefficients obtained were then standardized (divided by the variance) to become the path coefficients for the direct effects. The standardization corrected for differences in variability and metric among predictor variables, thus allowing direct comparison of effects of different types of variables. Indirect effects of predictor variables on SRHS were calculated from the direct effects by multiplying the appropriate path coefficients and using rules of covariance algebra. ${ }^{2}$ The total effects of a predictor variable on SRHS were the sum of the direct and indirect effects.

The modeling of utilization was more complex, however, because $81 \%$ of the study respondents did not have hospital stays, $18 \%$ did not have physician visits, and the distribution of use was skewed among those who had use. Therefore, in order to model the direct effects of predictor variables on utilization, we used two-part models as described by Duan and Manning. ${ }^{3}$ We evaluated the effects of predictor variables on the probability of use and then on the amount of use among those with use in order to derive the direct joint effects of predictors on the amount of use in the total population (users and nonusers). This model follows from Bayes's rule for joint probability, where

$$
E(Y)=E(Y \mid Y>0)^{*} \operatorname{pr}(Y>0) .
$$

A probit model was used to evaluate the probability of use as a function of the predictor variables in the total study sample. Because the probit coefficients obtained represent a nonlinear relationship between the dependent and predictor variables, these coefficients were transformed into marginal effects, representing the independent effect of a predictor variable on the dependent variable when the other variables are fixed at the sample mean. The sample mean for dummy variables is the proportion of respondents who are coded as "one." Also, in this model, the number of disabilities and SRHS are used as interval level variables. Therefore, the marginal effects represent a change in only one level of disability or SRHS.

The amount of use among users was evaluated by OLS regression on the log transformation of the amount of use using as regressors the same predictor variables. The marginal joint effects on the amount of use in the total sample were obtained by multiplying the marginal effects calculated from probit coefficients (probability of use) by the exponentiated OLS regression coefficients (amount of use, if any) and weighting the results by a correction factor based on the mean of the exponentiated residuals. ${ }^{3}$ Separate two-stage models were estimated for direct joint effects of physician visits and hospital stays.

In order to evaluate the direct and indirect effects of chronic diseases on hospital stays and physician visits in the total analytic sample, we used a variation of the method of Winship and Ware. ${ }^{21}$ In this technique, direct effects and total effects of predictor variables are estimated, and the indirect effects are the differences between the total and direct effects. In our analysis, the direct impacts of chronic diseases on utilization were measured by full models, two-stage models that included the intervening variables (as described in detail above). Figure 2 illustrates this model and the variables used; the intervening variables, disability and SRHS, are shaded. The total effects of specific chronic diseases were evaluated by estimating reduced models, which are the same two-stage models except that the intervening variables of disability and 
Figure 2. Figure 2 illustrates the conceptual models and variables used to evaluate the direct and indirect effects of chronic diseases on utilization. Disability and SRHS (shaded) are intervening variables. Age, gender, race, education and social isolation are control variables. The reduced model for the effects of chronic diseases on utilization was estimated by two-stage models, without including intervening variables, and thus represented the total effects. The full model for the effects of chronic diseases on utilization was estimated by two-stage models, including the intervening variables, and represented direct effects. Indirect effects are the differences between the total and direct effects and represent the effects of intervening variables.

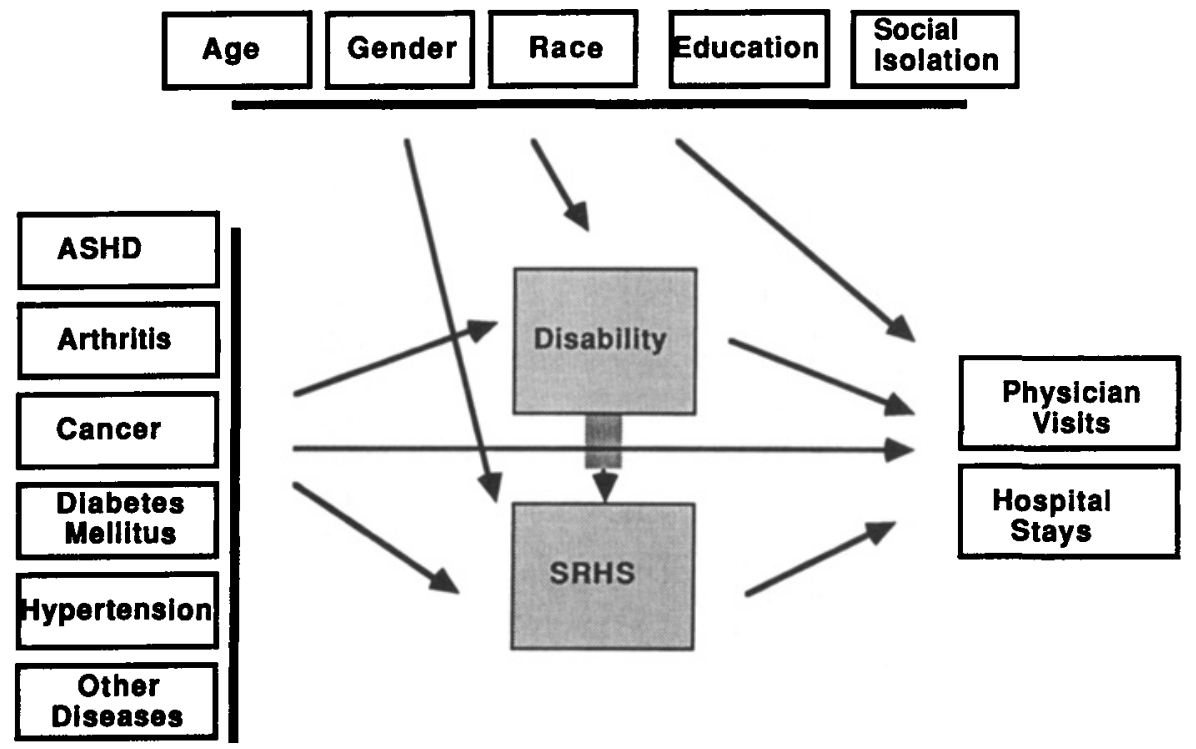

SRHS are left out. Subtracting the direct effects (joint effects estimated from the full model) from the total effects (joint effects estimated from the reduced models) gives the indirect effects attributed to the intervening variables. This method does not allow specification of the relationship between the intervening variables (shaded line in Figure 2). The statistical package used was SASpc. ${ }^{22}$

\section{RESULTS}

Table 2 shows results from the path analysis, or multiple equation OLS model, of the structure of physical health. The direct effects of specific chronic diseases on number of disabilities and SRHS are shown, as are the indirect and total effects of each chronic disease on SRHS. Arthritis and the presence of other comorbid diseases had large indirect effects on SRHS, equal to or greater than their direct effects on SRHS. Hypertension and cancer had small indirect effects, while diabetes and ASHD had substantial indirect effects, even though they were smaller than the direct effects. The largest total impact on SRHS was from disability, other comorbid diseases, arthritis, and ASHD.

Table 3 shows results from the two stage model for the direct effects of specific chronic diseases, disability, and SRHS on the expected amount of physician visits (Table 3a) and hospital stays (Table $3 \mathrm{~b}$ ) in the past year. The first column of Table $3 a$ gives the marginal effects (from the probit model) of the predictor variables on the probability of any physician visits. Results show that the strongest predictors of any physician visit were the presence of hypertension and diabetes mellitus, followed by cancer and ASHD. Therefore, if all other predictor variables were set at the sample mean, the presence of diabetes mellitus increased the probability of a physician visit by $9.7 \%$, while the presence of ASHD increased this probability by $7.4 \%$.

Table 2. Path analysis-Structure of physical health

\begin{tabular}{lcccc}
\hline Predictor Variable & $\begin{array}{c}\text { Disability } \\
\text { Direct Effects }\end{array}$ & $\begin{array}{c}\text { SRHS Direct } \\
\text { Effects }\end{array}$ & $\begin{array}{c}\text { SRHS Indirect } \\
\text { Effects }\end{array}$ & $\begin{array}{c}\text { SRHS Total } \\
\text { Effects }\end{array}$ \\
\hline ASHD & .0918 & .0967 & .0369 & .1336 \\
Arthritis & .1796 & .0768 & .0722 & .1490 \\
Cancer & .0268 & .0614 & .0108 & .0722 \\
Diabetes & .0991 & .0779 & .0398 & .1177 \\
Hypertension & .0491 & .0687 & .0197 & .0884 \\
Other diseases & .2430 & .0676 & .0977 & .1653 \\
Disability & & .4021 & & .4021 \\
Sample Size & 10227 & & & \\
$R^{2}$ & .2515 & 10227 & & \\
\hline
\end{tabular}

All path coefficients (standardized regression coefficients) shown are significant at $P<0.001$. 
Table 3. Direct effects of specific chronic diseases and health status on utilization

\begin{tabular}{|c|c|c|c|c|c|c|}
\hline \multirow[b]{2}{*}{ Predictor Variable } & \multicolumn{3}{|c|}{ Table 3a. Physician Visits } & \multicolumn{3}{|c|}{ Table 3b. Hospital Stays } \\
\hline & $\begin{array}{l}\text { Marginal } \\
\text { Effect on } \\
\text { Probability of } \\
\text { Use (probit } \\
\text { model) }\end{array}$ & $\begin{array}{l}\text { Multiplicative } \\
\text { Effect on } \\
\text { Amount of Use } \\
\text { (OLS model) }\end{array}$ & $\begin{array}{l}\text { Joint } \\
\text { Effect }\end{array}$ & $\begin{array}{l}\text { Marginal } \\
\text { Effect on } \\
\text { Probability of } \\
\text { Use (probit } \\
\text { model) }\end{array}$ & $\begin{array}{l}\text { Multiplicative } \\
\text { Effect on } \\
\text { Amount of Use } \\
\text { (OLS model) }\end{array}$ & $\begin{array}{l}\text { Joint } \\
\text { Effect }\end{array}$ \\
\hline ASHD & .0740 & 1.1825 & .1419 & .0771 & 1.130 & .0949 \\
\hline Arthritis & .0364 & 1.0669 & .0630 & NS & NS & \\
\hline Cancer & .0798 & 1.2065 & .1562 & .0748 & 1.0895 & .0901 \\
\hline Diabetes mellitus & .0966 & 1.2854 & .2014 & .0590 & NS & \\
\hline Hypertension & .1176 & 1.1270 & .2150 & .0139 & NS & \\
\hline Other disease & .0385 & 1.0845 & .0677 & .0634 & NS & \\
\hline Disability & .0053 & 1.0426 & .0064 & .0106 & 1.0125 & .0119 \\
\hline SRHS & .0285 & 1.1763 & .0544 & .0461 & 1.0567 & .0538 \\
\hline Sample size & 10228 & 8368 & 10228 & 10263 & 1983 & 10263 \\
\hline Correction factor & & 1.6219 & & & 1.1066 & \\
\hline Likelihood ratio & 1078.853 & & & 965.639 & & \\
\hline$R^{2}$ & & 175 & & & .093 & \\
\hline
\end{tabular}

All effects shown for probit and OLS models are significant at $P<0.001$.

The second column of Table 3a shows the results of the second part of the two-stage model, which used an OLS regression model to study predictor variable effects on the expected number of visits among those with visits. Because the dependent variable was the natural logarithm of the amount of visits, the exponentiated regression coefficients (mutliplicative effects) are shown in column two. Diabetes mellitus and cancer had the strongest effects. For example, for those who ever saw a physician in the last 12 months, the number of visits for those who had diabetes mellitus was .28 greater than for those who did not.

The final column in Table 3a displays the impact of the predictor variables on the number of physician visits among the total sample, including both users and nonusers. The number given represents the joint effects of the predictor variables on the expected number of physician visits for any individual in the sample. The joint effects again demonstrated the important impact of diabetes, hypertension, cancer, and ASHD and the minor impact of arthritis on utilization when other comorbidities were controlled. For example, the expected number of physician visits among those with diabetes mellitus was .20 higher than for those without, relative to the entire sample.

Table $3 \mathrm{~b}$ presents results of the two-stage model for hospital stays. The interpretations of the probit model's marginal effects, the OLS model's multliplicative effects, and the joint effects are the same. The presence of ASHD and cancer had the most effect on the probability of having had a hospital stay within the last 12 months. Arthritis had no significant impact. Among those with stays, however, only ASHD, cancer, SRHS, and number of disabilities had a significant impact on the number of stays. In the total analytic sample, ASHD and cancer had the most impact.

Table 4 shows the total, direct, and indirect joint effects of specific chronic diseases on the expected utilization in the total analytic sample. As the table columns indicate, the total effects are the joint effects calculated from the reduced model, the direct effects are the joint effects calculated from the full model, and the indirect effects are the differences between the total and direct joint effects (See Figure 2). For

Table 4. Indirect effects of health status on disease-specific utilization

\begin{tabular}{|c|c|c|c|c|c|c|}
\hline \multirow[b]{2}{*}{$\begin{array}{l}\text { Independent } \\
\text { Variable }\end{array}$} & \multicolumn{3}{|c|}{ Table 4a. Physician Visits } & \multicolumn{3}{|c|}{ Table 4b. Hospital Stays } \\
\hline & $\begin{array}{c}\text { Total Effects } \\
\text { (Reduced Model) }\end{array}$ & $\begin{array}{l}\text { Direct Effects } \\
\text { (Full Model) }\end{array}$ & $\begin{array}{c}\text { Indirect Effects } \\
\text { (Differences) }\end{array}$ & $\begin{array}{c}\text { Total Effects } \\
\text { (Reduced Model) }\end{array}$ & $\begin{array}{l}\text { Direct Effects } \\
\text { (Full Model) }\end{array}$ & $\begin{array}{c}\text { Indirect Effects } \\
\text { (Differences) }\end{array}$ \\
\hline ASHD & .2101 & .1419 & .0682 & .1566 & .0949 & .0617 \\
\hline Arthritis & .1158 & .0630 & .0528 & NS & NS & \\
\hline Cancer & .1932 & .1562 & .0370 & .1159 & .0901 & .0258 \\
\hline Diabetes mellitus & .2789 & .2014 & .0775 & $.1191^{\star}$ & NS & \\
\hline Hypertension & .2565 & .2150 & .0415 & NS & NS & \\
\hline Other diseases & .1331 & .0677 & .0654 & $.1298^{\star}$ & NS & \\
\hline
\end{tabular}

*Total effects represent joint effects significant at $P<0.02$ or less for both stages of the two-stage model. Otherwise, all effects shown were calculated from effects that were significant at $P<0.001$ at both stages of the two-stage model. 
physician visits (Table 4a) arthritis and comorbid diseases had indirect joint effects nearly as large as their direct effects. Diabetes and ASHD had substantial indirect effects, although these are smaller than the direct effects, while cancer and hypertension acted mainly through direct effects. Only cancer and ASHD impacted hospital stays in all models (Table 4b). ASHD had a relatively large indirect effect, while cancer again acted mainly directly. Diabetes and the presence of other comorbid diseases had borderline significant total effects on hospital stays, but insignificant direct effects, suggesting that their impact on hospital stays was mediated through their effects on physical health status.

\section{DISCUSSION}

This research emphasizes the consequences of chronic diseases on physical health status and health services utilization. We have shown how disability and SRHS mediated the relationship between chronic diseases and health services utilization and that this relationship was affected to a greater or lesser extent, depending on the particular chronic disease considered. Arthritis and other comorbid diseases had large effects on disability and, subsequently, on SRHS. Most effects of arthritis and comorbid diseases on utilization, therefore, arose from their effects on physical health. ASHD and diabetes mellitus had major effects on all outcomes and exhibited both direct and indirect impacts on utilization. Cancer and hypertension had little effect on disability and SRHS and exerted direct effects mainly on utilization.

Our research evaluates the impact of physical health status on health services utilization by viewing both as consequences of chronic diseases. The models presented in this paper considered the links to disability and SRHS from specific chronic diseases, thus capturing the complexity of physical health status. We then evaluated the impact of physical health status on utilization. In previous studies based on the HBM, disability and SRHS have often been linked to health services utilization, ${ }^{8-11}$ and SRHS has been used alone to predict health services utilization, including physician visits, ${ }^{23}$ hospital days, and admissions to nursing homes. ${ }^{24,}{ }^{25}$ However, the multidimensional aspect of physical health status has not generally been studied for its links to utilization.

We also evaluated the direct effects of specific chronic diseases on utilization. Previously, chronic diseases have been poorly operationalized as need variables in behavioral models of utilization, and specific chronic diseases have rarely been evaluated for their direct effects on utilization. In "medical models" of health service delivery, based on area variations of rates or types of medical or surgical procedures, ${ }^{26-28}$ medical diagnoses have figured more prominently. However, within the area variation models, ${ }^{29}$ patient level information is generally missing. ${ }^{30}$ Thus, these models cannot address sample specific disease and health status information among those who use or do not use specific health services. By using a disease-specific focus to study both physical health status and utilization, we have begun to bridge the conceptual gap between the patient focused, behavioral models of health services utilization, and the physician practice-centered, medical models of health services delivery.

Limitations of this study involve measurement issues concerning the independent variables and distribution and censoring problems involving the dependent variables. Be- cause this research focused on self-reported chronic diseases, it is important to consider measurement of chronic diseases and conditions in health interview surveys. This has been a recognized problem since the early days of the Health Interview Survey. ${ }^{31} \mathrm{~A}$ recent study found that in older adults, the comparability of self-report and medically diagnosed conditions varied by condition. ${ }^{32}$ In this study we did not analyze additional information about self-reported chronic conditions available in the SOA, such as duration of condition and cure or control of condition. Future analyses are planned to evaluate the possible contribution of these and similar questions to chronic disease measurement in health surveys and to adjust for measurement error in self-reported conditions. ${ }^{33,} 34$

There are also problems associated with measuring and conceptualizing disability in health interview surveys. Some authors consider disability to be hierarchical; ${ }^{4}$ others feel that IADL's may be closely related to cognitive function. ${ }^{17}$ Wolinsky and others have distinguished between upper body and lower body dysfunction. ${ }^{35}$ We feel that our approach is reasonable for this study, and we recognize that, as yet, there is no clear-cut method to model disability in all settings.

SRHS is also an ordinal variable that has been measured in several ways, sometimes as a single question, sometimes as a latent construct with several indicators. ${ }^{19}$ It has become the most commonly used measure of general health status assessment and performs well even as a single question. ${ }^{25}$

The distributional problems with measures of utilization, physician visits, and hospital stays in the past year are addressed by our use of the two-stage model. However, there is still an issue of limited dependent variables. Current nonusers may become users in the future. However, because the data specify use in the past 12 months, this conceptual problem is eliminated by design.

Finally, this study was based on cross sectional information from the SOA. This presents problems because the presence of certain conditions was ascertained for the preceding 30 days, while the presence of other conditions, disabilities, and utilization, was ascertained for the past year. Although modeling techniques used in this research are designed for cross-sectional analysis, longitudinal study will be important to understand the relationship between chronic diseases, physical health status, and utilization over time. However, longitudinal analysis has its own set of problems. ${ }^{36}$ It also depends strongly on theoretical specifications and simplifying assumptions. In longitudinal analysis, for example, transitions in conditions and disability can only be estimated once during a panel time period. Transitions from nonuser to user will be confounded by competing risks and complex probability assumptions, and measurement issues and variable distributions will assume more importance because decreasing sample sizes will force collapsing of categories. $^{37}$

We feel that our multiple equation approach has led to results with important implications for both clinical practice and research. The impact of chronic disease on physical health status is increasingly recognized as an important clinical concern and research area. ${ }^{38,} 39$ A disease-specific focus will be necessary to both understand and influence the trajectory from chronic disease to decreased health status. In addition, our research points out that physical health status is not only a medical outcome, but also a predictor of ambulatory and acute health services utilization. The dis- 
ease-specific component of physical health is crucial to understanding the role of health status in utilization, just as health status is crucial to understanding the role of specific diseases in utilization. Inserting a disease-specific focus into a behavioral model of utilization enriches the model conceptually and may enhance its usefulness for the study of health services utilization from the patient point of view.

\section{ACKNOWLEDGMENTS}

The authors wish to thank Dr. Jeffrey B. Halter and Dr. Linda Nyquist, for advice and suggestions in the preparation of this manuscript, and Ms. Judy Seeger for secretarial help.

\section{REFERENCES}

1. Rothenberg RB, Koplan JP. Chronic disease in the 1990s. Ann Rev Public Health 1990;11:267-96.

2. Duncan OD. Introduction to Structural Equation Models, New York: Academic Press, 1975.

3. Duan N, Manning WG, Morris $\mathrm{CN}$ et al. A comparison of alternative models for the demand for medical care. J Bus Econ Stat 1983;1:115-26.

4. Whitelaw NA, Liang J. The structure of the OARS physical health measures. Med Care 1991;29:332-47.

5. Anderson JG, Bartkus DE. Choice of medical care: A behavioral model of health and illness behavior. J Health Soc Behav 1973;14:348-62.

6. Hulka BS, Wheat JR. Patterns of utilization: The patient perspective. Med Care $1985 ; 23: 438-60$.

7. Wolinsky FD, Johnson RJ. The use of health services by older adults. J Gerontol 1991;46:S345-57.

8. Wan TH, Arling G. Differential use of health services among disabled elderly. Res Aging 1983;5:411-31.

9. Branch L, Jette A, Evashwick $\mathrm{C}$ et al. Toward understanding elders health service utilization. J Community Health 1981;7:80-92.

10. Coulton C, Frost AK. Use of social and health services by the elderly. J Health Soc Behav 1082:23:330-9.

11. Evashwick C, Rowe G, Diehr P et al. Factors explaining the use of health care services by the elderly. Health Serv Res 1984;19:357-82.

12. Fitti J, Kovar MG. The Supplement on Aging (SOA) to the 1984 National Health Interview Survey. PHHS Pub. No. (PHS)87-1323, Ser:1, No. 21. Washington, DC: US Government Printing Office, 1987.

13. Katz S, Akpom CA. A measure of primary sociobiological functions. Int J Health Serv 1976;6:493-510.

14. Lawton MP, Brody EM. Assessment of older people: Self-maintaining and instrumental activities of daily living. Gerontologist 1969;9:179-86.

15. Rogers RG, Rogers A, Belanger A. Disability-free life among the elderly in the United States. J Aging Health 1992;4:19-42.

16. Manton KG. Epidemiological, demographic and social correlates of diasbility among the elderly. Milbank Q 1989;67S2:13-58.

17. Verbrugge LM. Disability. Rheum Dis Clin North Am 1990;16:741-61.
18. Verbrugge LM, Gates DM, lke RW. Risk factors for disability among U.S. adults with arthritis. J Clin Epidemiol 1991;44:167-82.

19. Liang J. Self-reported physical health among aged adults. J Gerontol $1985 ; 41: 248-60$.

20. Crimmins EM, Saito Y. Getting better and getting worse. J Aging Health $1993 ; 5: 3-36$

21. Winship C, Mare RD. Structural equations and path analysis for discrete data. Am J Soc 1983;89:54-110.

22. SAS Institute. SAS Language Guide for Personal Computers, Release 6:03. Cary, NC: SAS Institute, 1968.

23. LaRue A, Bank B, Jarvik L et al. Health in old age: How do physicians' ratings and self ratings compare? J Gerontol 1979;34:687-91.

24. Linn BS, Linn MW. Objective and self-assessed health in the old and very old. Soc Sci Med 1980;14a:311-315.

25. Weinberger M, Darnell JC, Tierney WM et al. Self-rated health as a predictor of hospital admission and nursing home placement in elderly public housing tenants. Am J Public Health 1986;76:457-9.

26. Brook RH, Park RD, Chassin RM et al. Predicting appropriate use of carotid endarterectomy, upper gastrointestinal endoscopy and coronary angiography. N Engl J Med 1990;323.

27. Chassin MR, Kosecoff J, Park RE et al. Does inappropriate use explain geographic variation in the use of health care services? A study of three procedures. JAMA 1987;258:2533-7.

28. Wennberg JE, Freeman JL, Culp WJ. Are hospital services rationed in New Haven or over-utilized in Boston? Lancet 1987;1:1185-9.

29. Wennberg JE, Gittlesohn A. Amall area variation analysis in health care delivery. Science 1973;182:1102-8.

30. Longo DR. Patient practice variation: A call for research. Medical Care 1993;31:YS81-5.

31. Madow WG. Net differences in interview data on chronic conditions and information derived from medical records in Vital Health Stat. Washington, DC: US Government Printing Office, 1973.

32. Ford $\mathrm{AB}$ et al. Health and function in the old and very old. J Am Geriatr Soc 1988;36:187-97.

33. Joreskog KG, Sorbom D. LISREL. Analysis of Linear Structural Relationships by the Method of Maximum Likelihood, 4th Ed. Morresville, IN: Scientific Software, Inc, 1986.

34. Bollen KA. Structural Equations with Latent Variables. Wiley Series, Probability and Mathematical Statistics. New York: John Wiley and Sons, 1989.

35. Wolinsky FD, Coe RM, Miller DK et al. Measurement of the global and functional dimensions of health status in the elderly. J Gerontol 1984;39:88-92.

36. Menard S. Longitudinal Research. In: MS Lewis-Beck, ed. Quantitative Applications in the Social Sciences. Newbury Park, CA: Sage Publications, 1991.

37. Kessler RC, Greenberg DF. Linear Panel Analysis: Models of Quantitative Change. New York: John Wiley, 1981.

38. Stewart AL, Greenfield S, Hays RD et al. Functional status and well-being of patients with chronic conditions. JAMA 1989;262:907-13.

39. Tarlov AR, Ware JE, Greenfield S et al. The medical outcomes study: An application of methods for monitoring the results of medical care. JAMA 1989;262:925-30. 